\title{
Importance of CT-scan predicting clinical outcomes in gastro- intestinal perforation
}

\author{
Roberto Bini ${ }^{1}$, Clemente Ronchetta ${ }^{2}$, Silvia Picotto ${ }^{2}$, Gitana Scozzari ${ }^{3}$, Shailvi Gupta ${ }^{4}$, Simone Frassini ${ }^{1}$, \\ Osvaldo Chiara ${ }^{1}$
}

${ }^{1}$ University of Milan, General Surgery and Trauma Team, ASST Grande Ospedale Metropolitano Niguarda, Milan, Italy; ${ }^{2}$ General Surgery Unit, Ospedale San Giovanni Bosco, Turin, Italy; ${ }^{3} \mathrm{AOU}$ Città della Salute e della Scienza di Torino, Turin, Italy; ${ }^{4} \mathrm{R}$ Adams Cowley Shock Trauma Center, University of Maryland, Baltimore, MD, USA

Contributions: (I) Conception and design: R Bini, C Ronchetta, S Picotto, G Scozzari, S Frassini; (II) Administrative support: R Bini, O Chiara; (III) Provision of study materials or patients: R Bini, C Ronchetta, S Picotto, G Scozzari; (IV) Collection and assembly of data: R Bini, C Ronchetta, S Picotto, G Scozzari; (V) Data analysis and interpretation: R Bini, C Ronchetta, S Picotto, G Scozzari; (VI) Manuscript writing: All authors; (VII) Final approval of manuscript: All authors.

Correspondence to: Roberto Bini, MD. University of Milan, General Surgery and Trauma Team, ASST Grande Ospedale Metropolitano Niguarda, Milano, Piazza Ospedale Maggiore 3, 20162 Milan, Italy. Email: re.bini@libero.it.

Background: Gastrointestinal (GI) perforations are an acute surgical dilemma, with diagnostic workup often requiring abdominal imaging. Post-operative care for these patients may vary and oftentimes includes ICU care for sepsis, but not always. We evaluated if free fluid and air on computed tomography (CT) could be associated with sepsis, septic shock and mortality in GI perforations. The aim of our study was a correlation between a new CT-scan scoring system and septic complications in GI perforations.

Methods: We conducted an observational retrospective study about patients who underwent emergency surgery for intestinal perforation between January 2014 and June 2017. Inclusion criteria were a CTscan positive for free fluid and air, and an intestinal perforation confirmed intraoperatively. A CT-score was created to evaluate location and extent of free fluid and air related to clinical outcome and prognosis. Univariate analysis between the CT score and the various clinical outcomes was conducted with the nonparametric Mann-Whitney test for continuous variables and with the chi-square test for categorical variables. Results: One-hundred and fifty-one patients were evaluated. The mortality was $23.18 \%$ and the complications were present in $45.95 \%$. The median CT score for patients who developed complications was 3 , compared with a value of 2 in the absence of complications $(\mathrm{P}=0.008)$. A CT score of 4 or greater had a sensitivity and specificity for predicting pre-operative sepsis of $73.33 \%$ and $64.42 \%$ respectively, and for preoperative septic shock of $35.56 \%$ and $93.27 \%$. Looking at post-operative sepsis, sensitivity and specificity were $57.45 \%$ and $70.19 \%$, and for septic shock $36.17 \%$ and $85.85 \%$. Spearman correlation analysis revealed that at higher scores at CT score corresponded higher scores at the P-POSSUM morbidity, P-POSSUM mortality and WSES Sepsis Severity Score.

Conclusions: Our CT score shows a significant correlation with validated predictive scoring systems with regards to predicting sepsis, septic shock and complications-and seems to be a useful outcome predictor in GI perforation.

Keywords: Computed tomography-scan (CT-scan); gastro-intestinal perforation; emergency surgery

Submitted Mar 04, 2020. Accepted for publication Aug 28, 2020.

doi: $10.21037 / \mathrm{atm}-20-2184$

View this article at: http://dx.doi.org/10.21037/atm-20-2184 


\section{Introduction}

Gastrointestinal (GI) perforations may be suspected based upon history and physical examination findings, but oftentimes a diagnosis relies upon abdominal imaging studies, typically abdominal computed tomography (CT) images (1). These images may show extraluminal air within the peritoneal cavity, retroperitoneum, GI fistula formation, intra-abdominal or mediastinal abscess or free fluid (2). Initial management includes intravenous fluid therapy and antibiotic administration though many patients require urgent surgical intervention for abdominal source control. The presence of abdominal sepsis and septic shock affect the severity of the GI perforation and may lead to a poorer prognosis. An accurate risk prediction score is desirable, as both mortality and morbidity often drive the decision of a high-risk surgery. Scoring systems evaluating risk of death and complications from bowel perforations focus primarily upon post-operative mortality and morbidity and sepsis severity (3). The Physiological and Operative Severity Score for the enumeration of Mortality and morbidity (POSSUM) and its variant Portsmouth POSSUM (P-POSSUM) consider 12 physiological and six operative variables, in order to predict postoperative morbidity and 30-day mortality (4). The World Society of Emergency Surgery (WSES) Sepsis Severity Score is a practical clinical severity score for patients with complicated intra-abdominal infection that evaluate clinical conditions at admission (severe sepsis/septic shock), the origin of the infection, delay in source control, the setting of acquisition and any risk factors such as age and immunosuppression (5). However, both POSSUM and WSES Sepsis Severity Score require parameters not available pre-operatively and are not widely used in clinical practice when a GI perforation is suspected.

To our knowledge, none of the scores used in GI perforations take into consideration the use of radiological data as a prognostic factor, despite its wide use and high accuracy in the early diagnostic stages.

The purpose of our study was to determine whether CT findings in GI perforations are helpful in predicting clinical outcomes compared to P-POSSUM and WSES Sepsis Severity Score. We evaluated if free fluid or free air on CT could be associated with sepsis, septic shock or mortality in GI perforations. The aim was to introduce a new scoring system based on CT findings as an early prognostic tool.

We present the following article in accordance with STROBE reporting checklist (available at http://dx.doi. org/10.21037/atm-20-2184).

\section{Methods}

\section{Patients}

We reviewed observationally the medical records of patients (age $>18$ years) who underwent emergency surgery for intestinal perforation in S.G. Bosco Hospital, Turin, between January 2014 and June 2017. Patients were identified according to the International Classification of Diseases, Clinical Modification Code for intestinal perforations (ICD-9-CM 569.83, ICD-10-CM K63.1). In order to be included in the study, patients with GI perforation had to be diagnosed by detection of free intraperitoneal fluid and air on CT and the diagnosis had to be confirmed intraoperatively. Exclusion criteria were unavailable clinical and radiological data and GI perforations diagnosed only intraoperatively. All imaging, laboratory and clinical data were evaluated (Figure 1).

\section{Imaging data}

The pre-operative CT images were interpreted by two independent reviewers without knowledge of the patient characteristics and clinical outcome. When the CT findings observed were inconsistent or not in agreement, a definitive result was reached by consensus. For every patient, CT findings were recorded and subdivided according to the location of free intraperitoneal fluid $(>4 \mathrm{~mL})$ and/or gas [upper left quadrant (ULQ), upper right quadrant (URQ), lower left quadrant (LLQ), lower right quadrant (LRQ), pelvis, inter-loops].

\section{CT score}

According to these results, a CT score was created in order to evaluate location and extent of free fluid and air related to clinical outcome and prognosis.

\section{Laboratory and clinical data}

Preoperative parameters were detected at admission or, in case of hospitalized patients, the last laboratory and clinical data prior to surgery. Sepsis and septic shock were assessed according to the "Surviving Sepsis Campaign: International Guidelines for Management of Sepsis and Septic Shock: 


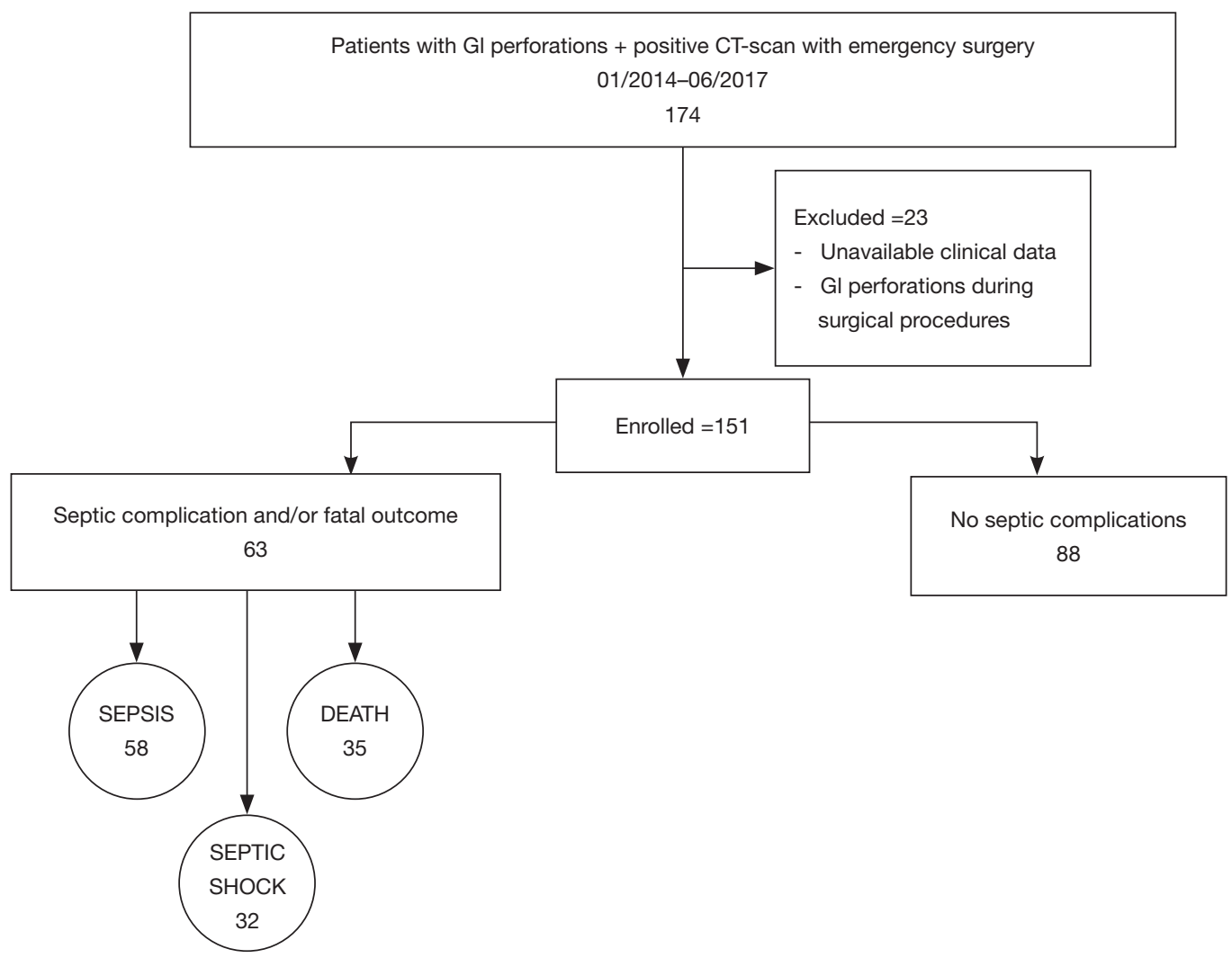

Figure 1 Flow chart showing patients included in the study and postoperative outcomes.

2016". WSES Sepsis Severity Score and P-POSSUM scores were assessed retrospectively (6). In our series we defined 'pre-operative' sepsis or septic shock as clinical conditions at the moment of CT-scan before any surgical treatment. "Post-operative" was a condition the patient developed after surgical treatment or that persisted despite operative intervention.

\section{Statistical analysis}

The categorical variables were reported as frequencies and percentages. The continuous variables were reported as averages and standard deviations (SD) for those with normal distribution and as median and interquartile range (IQR) for those with non-normal distribution. The normality of the distribution was verified with the ShapiroWilk test. Univariate analysis between the CT score and the various clinical outcomes was conducted with the nonparametric Mann-Whitney test for continuous variables and with the chi-square test for categorical variables (with Fisher correction when indicated). Spearman correlation was performed to evaluate the relationship between the CT score and the clinical and demographic data.

For each outcome a cut-off value was identified based on sensitivity, specificity, positive likelihood ratio (LR+) and negative likelihood ratio (LR-) analysis.

All tests were conducted in two queues, and the level of statistical significance was set at the conventional value of $\mathrm{P}<0.05$. The results were analyzed using Stata 13 statistical software (Stata Corp., College Station, TX).

The study was conducted in accordance with the Declaration of Helsinki (as revised in 2013). Our study protocol was reviewed and approved by ethics committee of San Giovanni Bosco Hospital, Turin, Italy (No. CE150089). Written informed consent was obtained from all subjects in our study.

\section{Results}

\section{CT score}

The CT-score results show that CT findings in the upper 


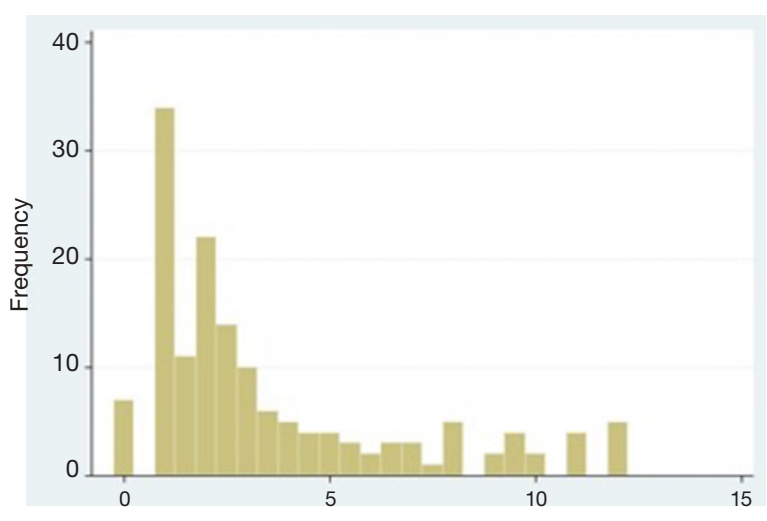

Figure 2 Distribution of the CT score.

right and left quadrants (URQ, ULQ) showed a stronger correlation with mortality, sepsis and septic shock, and were therefore assessed 3 points each. Since lower quadrants (LRQ, LLQ) were weakly associated to the clinical outcomes, they were assessed 1.5 point each. Free fluid in pelvis or inter-loops and free air were set to 1 point each, to a total of 12 points. The distribution of the CT score is shown in Figure 2.

\section{Patient characteristics}

Clinical characteristics of the entire cohort are shown in Table 1.

Of the 151 GI perforation considered, 128 (84.77\%) were primary perforations and $23(15.23 \%)$ were secondary to anastomotic dehiscence. The mortality rate was $23.18 \%$ (35 patients) and the complication rate was $45.03 \%$ (68 patients). Furthermore, 58 patients (38.41\%) suffered from postoperative sepsis and 32 patients $(21.19 \%)$ from septic shock. P-POSSUM mortality, P-POSSUM morbidity and WSES Sepsis Severity Score were assessed retrospectively. The median values were 7.3 (IQR 3.2; 21.2), 74.3 (IQR $55.7 ; 90.9$ ) and 4 (IQR 2; 7), respectively.

CT findings were divided according to location, as shown in Table 2.

Free abdominal fluid was mostly located in pelvis $(50.33 \%)$ and inter-loops (39.07\%), followed by lower quadrants (LRQ $32.45 \%$, LLQ $23.18 \%$ ). Free air was detected in more than half the cases (56.29\%).

Of the 36 patients $(23.84 \%)$ showing free fluid in the
Table 1 Clinical characteristics

\begin{tabular}{|c|c|}
\hline Characteristic & Number \\
\hline \multicolumn{2}{|l|}{ Sex } \\
\hline Female & $75(49.67 \%)$ \\
\hline Male & $76(50.33 \%)$ \\
\hline Median age in years [range] & $71[25-94]$ \\
\hline \multicolumn{2}{|l|}{ ASA score } \\
\hline ASA I & $3.31 \%$ \\
\hline ASA II & $24.50 \%$ \\
\hline ASA III & $49.01 \%$ \\
\hline ASA IV & $20.53 \%$ \\
\hline ASA V & $2.65 \%$ \\
\hline \multicolumn{2}{|l|}{ Perforation } \\
\hline Primitive & $128(84.77 \%)$ \\
\hline Secondary to dehiscence & $23(15.23 \%)$ \\
\hline \multicolumn{2}{|l|}{ GI tract affected } \\
\hline Gastroduodenal tract & $15(9.93 \%)$ \\
\hline Ileum & $34(22.52 \%)$ \\
\hline Appendix, cecum & $8(5.3 \%)$ \\
\hline Colon, rectum & $89(58.94 \%)$ \\
\hline Complication & $49(32.45 \%)$ \\
\hline Dehiscence, fistulae, abscess & 12 \\
\hline $\begin{array}{l}\text { ARDS, respiratory failure, AF, myocardial } \\
\text { infarction }\end{array}$ & 10 \\
\hline Others & 8 \\
\hline Sepsis & $58(38.41 \%)$ \\
\hline Septic shock & $32(21.19 \%)$ \\
\hline Deaths & $35(23.18 \%)$ \\
\hline Time from diagnosis to OR [IQR], hours & $5.15[3.1 ; 19]$ \\
\hline P-POSSUM morbidity [IQR] & $74.3[55.7 ; 90.9]$ \\
\hline P-POSSUM mortality [IQR] & $7.3[3.2 ; 21.2]$ \\
\hline WSES Sepsis Severity Score $(0-18)[\mathrm{IQR}]$ & $4[2 ; 7]$ \\
\hline ICU admission [IQR] & $44(29.14 \%)$ \\
\hline Duration of hospital stay in days [median] & $11[8 ; 17]$ \\
\hline
\end{tabular}

AF, atrial fibrillation; ASA, America Society of Anesthesiologists score; GI, gastrointestinal; OR, operating room. 
URQ at CT, 21 (58.33\%) developed sepsis compared to 37 septic patients $(32.17 \%)$ without URQ findings ( $\mathrm{P}=0.005)$; 14 patients $(38.89 \%)$ developed septic shock while showing free fluid in URQ, compared to the 18 patients (15.65\%) with septic shock and no URQ findings $(\mathrm{P}=0.003)$. Upper left free fluid was found in 27 patients (17.88\%), of which

Table 2 CT finding according to location

\begin{tabular}{lll}
\hline Location & No. of patients & $\%$ \\
\hline URQ & 36 & 23.84 \\
ULQ & 27 & 17.88 \\
LRQ & 49 & 32.45 \\
LLQ & 35 & 23.18 \\
Pelvis & 76 & 50.33 \\
Inter-loops & 59 & 39.07 \\
Free air & 85 & 56.29 \\
\hline
\end{tabular}

URQ, upper right quadrant; ULQ, upper left quadrant; LRQ, lower right quadrant; LLQ, lower left quadrant.
$16(59.26 \%)$ showed signs of sepsis against 42 septic patients $(33.87 \%)$ without ULQ findings $(\mathrm{P}=0.014)$. Septic shock was detected in $11(40.74 \%)$ patients presenting with ULQ findings and only in 21 (16.94\%) with negative CT scans $(\mathrm{P}=0.06)$ of the entire population. CT findings were associated with higher mortality only in perforations not due to anastomotic dehiscence. In particular, death occurred in 11 patients of $30(36.67 \%)$ with URQ findings and in 17 of $98(17.35 \%)$ without URQ findings ( $\mathrm{P}=0.025)$; in 9 patients of $23(39.13 \%)$ with ULQ findings and in 19 of 105 patients $(18.09 \%)$ without ULQ findings $(\mathrm{P}=0.027)$. No difference between the groups with or without free fluid or pneumoperitoneum were identified (Table 3).

\section{CT score compared to other predicting scores}

Spearman correlation analysis revealed that higher CT scores corresponded to higher scores at the P-POSSUM morbidity ( $\mathrm{rs}=0.24, \mathrm{P}=0.003$ ), P-POSSUM mortality (rs $=0.22, \mathrm{P}=0.007)$ and WSES Sepsis Severity Score $(\mathrm{rs}=0.18$, $\mathrm{P}=0.031)$ score (Table 4).

Table 3 Univariate analysis between CT score and outcomes (sepsis, septic shock, death) according to abdominal quadrant (chi-square test for categorical variables)

\begin{tabular}{|c|c|c|c|c|}
\hline Abdominal quadrants & Outcome & Patients with CT findings & Patients without CT findings & $P$ value \\
\hline URQ & Septic shock & 14/36 (38.89\%) & 18/115 (15.65\%) & 0.003 \\
\hline URQ primitive perforation & Death & $11 / 30(36.67 \%)$ & $17 / 98(17.35 \%)$ & 0.025 \\
\hline ULQ & Sepsis & 16/27 (59.26\%) & 42/124 (33.87\%) & 0.014 \\
\hline ULQ primitive perforation & Death & 9/23 (39.13\%) & 19/105 (18.09\%) & 0.027 \\
\hline \multirow[t]{2}{*}{ LRQ } & Sepsis & $22 / 49(44.90 \%)$ & 36/102 (35.29\%) & 0.256 \\
\hline & Septic shock & $14 / 49(28.57 \%)$ & 18/102 (17.65\%) & 0.124 \\
\hline LLQ & Sepsis & $12 / 35(34.29 \%)$ & 46/116 (39.65\%) & 0.567 \\
\hline Pelvis & Septic shock & $17 / 76(22.37 \%)$ & $15 / 75(20.00 \%)$ & 0.722 \\
\hline \multirow[t]{2}{*}{ Inter-loops } & Sepsis & 23/59 (38.98\%) & 35/92 (38.04\%) & 0.908 \\
\hline & Septic shock & $16 / 59(27.12 \%)$ & 16/92 (17.39\%) & 0.154 \\
\hline \multirow[t]{2}{*}{ Free air } & Sepsis & $35 / 85(41.18 \%)$ & 23/66 (34.85\%) & 0.428 \\
\hline & Septic shock & 16/85 (18.82\%) & $16 / 66(24.24 \%)$ & 0.419 \\
\hline
\end{tabular}

URQ, upper right quadrant; ULQ, upper left quadrant; LRQ, lower right quadrant; LLQ, lower left quadrant. 
Table 4 Spearman's correlation between CT score and other predicting scores

\begin{tabular}{lcc}
\hline \multirow{2}{*}{ Predicting scores } & \multicolumn{2}{c}{ CT score } \\
\cline { 2 - 3 } & $\mathrm{r}_{\mathrm{s}}$ & $\mathrm{P}$ \\
\hline WSES Sepsis Severity Score & 0.18 & 0.031 \\
Possum morbidity & 0.24 & 0.003 \\
Possum mortality & 0.22 & 0.007 \\
\hline
\end{tabular}

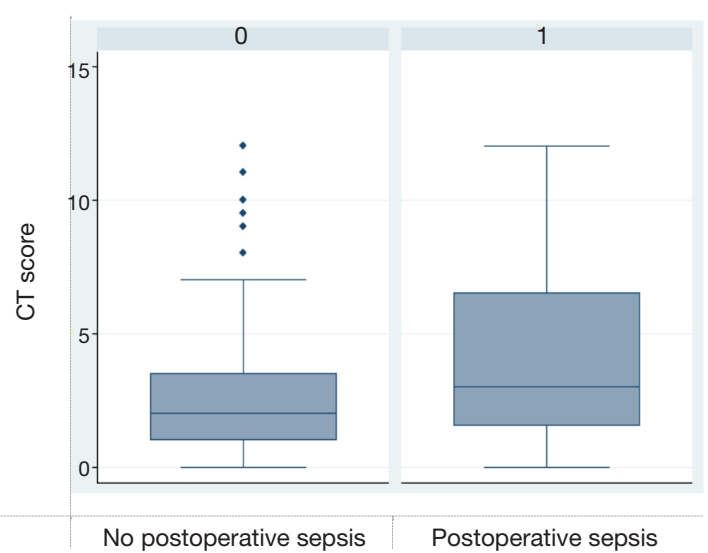

Figure 3 Chi-square test between CT score and postoperative sepsis.

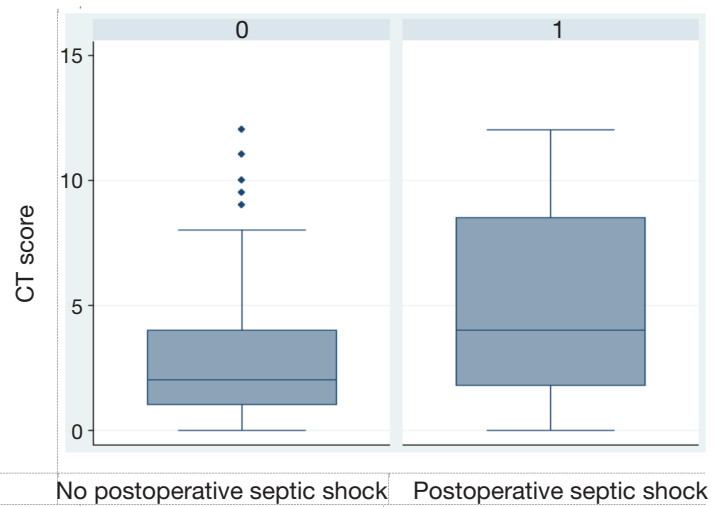

Figure 4 Chi-square test between CT score and postoperative septic shock.

\section{CT score and outcomes}

Patients presenting with sepsis had a CT score median of 3 , with a statistically significant difference from the group without postoperative sepsis $(\mathrm{P}=0.019)$. For the 32 patients diagnosed with septic shock $(21.19 \%)$, the median CT score was 4 , compared to the score of 2 for patients who did not develop septic shock ( $\mathrm{P}=0.014)$ (Figures 3,4).

The median CT score for patients who developed complications was 3 , compared to a value of 2 in the absence of complications $(\mathrm{P}=0.008)$. The difference in the CT score between deceased and not deceased patients was not statistically relevant (median score of 3 against 2; $\mathrm{P}=0.094$ ).

The AUCs (area under the curve) of the ability of CT score to predict pre-operative sepsis and septic shock were $0.610(\mathrm{P}=0.001,95 \% \mathrm{CI}, 0.515-0.697)$ and $0.682(\mathrm{P}=0.001$, 95\% CI, 0.545-0.818), respectively.

A CT score of 4 or greater had a sensitivity and specificity for predicting pre-operative sepsis of $73.33 \%$ (95\% CI, 58.06-85.40) and 64.42\% (95\% CI, 54.43-73.57) respectively, and for predicting pre-operative septic shock of $35.56 \%$ (95\% CI, 21.87-51.22) and $93.27 \%$ (95\% CI, $86.62-97.25)$, respectively, and was therefore selected as the cutoff value to identify high-risk patients. In pre-operative sepsis, the positive predictive value (PPV) was $47.14 \%$ (95\% CI, 39.48-54.95) and negative predictive value (NPV) 84.81 (95\% CI, 77.11-90.25). Positive Likelihood ratio was 2.06 (95\% CI, 1.51-2.83). In pre-operative septic shock PPV was 69.57\% (95\% CI, 50.26-83.80) and NPV 76.98\% (95\% CI, 72.80-80.70). Positive Likelihood ratio was 5.28 (95\% CI, 2.33-11.95).

A CT score of 4 or greater had a sensitivity and specificity for predicting post-operative sepsis of $57.45 \%$ (95\% CI, 42.18-71.74) and 70.19\% (95\% CI, 60.42-78.77) respectively, and for predicting post-operative septic shock of $36.17 \%$ (95\% CI, 22.67-51.48) and $85.58 \%$ (95\% CI, 77.33-91.70), respectively. In post-operative sepsis PPV was 46.55\% (95\% CI, 37.23-56.12) and NPV 78.49\% (95\% CI, 71.90-83.89). Positive Likelihood ratio was 2.01 (95\% CI, $1.67-2.93)$.

In post-operative septic shock PPV was 52.12\% (95\% CI, 38.28-67.44) and NPV 74.79\% (95\% CI, 70.23-78.86). Positive Likelihood ratio was 2.51 (95\% CI, 1.37-4.58).

The AUCs of the ability of CT score to predict complications was 0.618 ( $\mathrm{P}=0.003,95 \% \mathrm{CI}, 0.516-0.718)$. A CT score of 4 or greater has a sensitivity and specificity for predicting complications of $64.44 \%$ (95\% CI, 48.78-78.13) and $62.86 \%$ (95\% CI, 52.88-72.09) respectively. PPV was $42.65 \%$ (95\% CI, 34.83-50.85) and NPV 80.49\% (95\% CI, 73.05-86.26). Positive likelihood ratio was 1.86 (95\% CI, 1.2-2.6) (Table 5).

\section{Discussion}

GI perforations can be due to a variety of etiologies 
Table 5 Statistic about CT score and outcomes

\begin{tabular}{|c|c|c|c|c|c|}
\hline Variable & $\mathrm{P}$ & $\begin{array}{c}\text { Positive predictive value } \\
\text { (PPV) }\end{array}$ & $\begin{array}{c}\text { Negative predictive value } \\
\text { (NPV) }\end{array}$ & Sensitivity & Specificity \\
\hline Sepsis pre & 0.001 & $47.14 \%$ & $84.81 \%$ & $73.33 \%$ & $64.42 \%$ \\
\hline Sepsis post & 0.001 & $46.55 \%$ & $78.49 \%$ & $57.45 \%$ & $70.19 \%$ \\
\hline
\end{tabular}

and may present a wide range of clinical manifestations, depending on the organ affected and on the nature of the contents released (air, succus, stool), as well as the ability of the surrounding tissues to confine those contents. Signs of perforation of the bowel wall, contrast extravasation, abscess, GI fistula, free air or fluid may be detected on CT scans. Initial management includes a careful evaluation of the patient status, regarding location and extent of the perforation, presence and severity of infection and hemodynamic stability. An early prognostic assessment is crucial to estimate the severity of the disease and to help decide the aggressiveness of treatment. These decision points may be directed using different scoring systems: POSSUM and P-POSSUM predict postoperative morbidity and 30-day mortality, and the WSES Sepsis Severity Score for patients with complicated intra-abdominal infections. However, none of these is specific for GI perforations nor take into consideration the site and extent of the perforation using radiographic imaging. Moreover, CT has been evaluated for predicting organ dysfunction and patients' survival in other clinical conditions. In 2007, De Waele et al. (7) developed a new un-enhanced CT scoring system called the extrapancreatic inflammation on CT (EPIC) score, and they concluded that this scoring system allowed accurate estimation of acute pancreatitis' severity and mortality within 24 hours of admission. In 2017, Chen et al. (8) evaluated the utility of the EPIC score for predicting organ failure in the early phase of acute pancreatitis as defined by the revised Atlanta classification, reporting a similar to higher accuracy in predicting the occurrence of organ failure in the early phase of acute pancreatitis compared to conventional scoring systems. In 2013, Stelter et al. (9) evaluated CT findings in septic patients with acute respiratory distress syndrome, grading findings on a 6-point scale. Patients with fatal outcome were reported to show a significantly higher CT score than survivors, with more ground-glass opacities and traction bronchiectasis.

A greater extension of free air or fluids, leading to a higher CT score, was detected in patients presenting with signs of postoperative sepsis or septic shock. In our series, fluid distribution in upper quadrants showed a strong correlation with outcomes: we are convinced this could be correlated to an anatomical and physiological tendency of fluid-when copious in severe GI perforationsaccumulating in those quadrants in supine position, such as during CT-scan exam. In turn, septic conditions correlate with a poorer prognosis, longer hospitalization and need for more aggressive management and surgery.

Furthermore, patients presenting with high scores at P-POSSUM and WSES Sepsis Severity Score also showed more complicated CT findings, with wider leakage and more abdominal quadrants involved. In these cases, CT scores higher than 4 could be clearly associated with poorer prognosis, in terms of post-operative sepsis and septic shock. Although a well-known sign in GI perforation, abdominal free air in our series was not significantly related to outcome. We think this is due to the small sample size and to the way free air was classified. Further studies are needed to clarify how free air should be integrated in a score like the one we are proposing.

Our study has several limitations. First, it was a nonrandomized retrospective study with a small study population. A sample of 151 patients could not be split into sub-samples without avoiding wide confidence intervals or risks of errors in statistical hypothesis testing. Second, we did not evaluate the etiology of the perforation in relation to the location of the CT findings. In particular, perforations secondary to anastomotic dehiscence, representing only the $15.23 \%$ of our cohort population, could not be studied separately, in spite of more complex data imaging and more severe clinical conditions. However, to our knowledge, this is the first study to test the relationship between CT 
findings, septic complications and scoring systems.

\section{Conclusions}

Free intraperitoneal fluid and gas on CT showed a significant correlation with a patient's clinical status and, above all, with internationally validated predictive scoring systems, such as the P-POSSUM and WSES Sepsis Severity Score. Furthermore, a CT score higher than 4 could be clearly associated with poorer prognosis, in terms of preand post-operative sepsis, septic shock and complications. To conclude, CT scan seems to be a useful outcome predictor in GI perforation and, should that be confirmed by future prospective studies, it should be included in the risk stratification.

\section{Acknowledgments}

Funding: None.

\section{Footnote}

Reporting Checklist: The authors present the study in accordance with the STROBE reporting checklist. Available at http://dx.doi.org/10.21037/atm-20-2184

Data Sharing Statement: Available at http://dx.doi. org/10.21037/atm-20-2184

Conflicts of Interest: All authors have completed the ICMJE uniform disclosure form (available at http://dx.doi. org/10.21037/atm-20-2184). The authors have no conflicts of interest to declare.

Ethical Statement: The authors are accountable for all aspects of the work in ensuring that questions related to the accuracy or integrity of any part of the work are appropriately investigated and resolved. The study was conducted in accordance with the Declaration of Helsinki (as revised in 2013). Our study protocol was reviewed and approved by ethics committee of San Giovanni Bosco Hospital, Turin, Italy (No. CE150089). Written informed consent was obtained from all subjects in our study.

Open Access Statement: This is an Open Access article distributed in accordance with the Creative Commons Attribution-NonCommercial-NoDerivs 4.0 International License (CC BY-NC-ND 4.0), which permits the non- commercial replication and distribution of the article with the strict proviso that no changes or edits are made and the original work is properly cited (including links to both the formal publication through the relevant DOI and the license). See: https://creativecommons.org/licenses/by-nc-nd/4.0/.

\section{References}

1. Faggian A, Berritto D, Iacobellis F, et al. Imaging Patients With Alimentary Tract Perforation: Literature Review. Semin Ultrasound CT MR 2016;37:66-9.

2. Del Gaizo AJ, Lall C, Allen BC, et al. From esophagus to rectum: a comprehensive review of alimentary tract perforations at computed tomography. Abdom Imaging 2014;39:802-23.

3. Nachiappan M, Litake MM. Scoring Systems for Outcome Prediction of Patients with Perforation Peritonitis. J Clin Diagn Res 2016;10:PC01-5.

4. Leung E, McArdle K, Wong LS. Risk-adjusted scoring systems in colorectal surgery. Int J Surg 2011;9:130-5.

5. Sartelli M, Abu-Zidan FM, Catena F, et al. Global validation of the WSES Sepsis Severity Score for patients with complicated intra-abdominal infections: a prospective multicentre study (WISS Study). World J Emerg Surg 2015;10:61.

6. Singer M, Deutschman CS, Seymour CW, et al. The Third International Consensus Definitions for Sepsis and Septic Shock (Sepsis-3). JAMA 2016;315:801-10.

7. De Waele JJ, Delrue L, Hoste EA, et al. Extrapancreatic inflammation on abdominal computed tomography as an early predictor of disease severity in acute pancreatitis: evaluation of a new scoring system. Pancreas 2007;34:185-90.

8. Chen $\mathrm{C}$, Huang $\mathrm{Z}, \mathrm{Li} \mathrm{H}$, et al. Evaluation of extrapancreatic inflammation on abdominal computed tomography as an early predictor of organ failure in acute pancreatitis as defined by the revised Atlanta classification. Medicine (Baltimore) 2017;96:e6517.

9. Stelter L, Steffen I, Pinkernelle JG, et al. Computed tomography findings in septic patients with acute respiratory distress syndrome: correlation with survival and pulmonary versus extrapulmonary septic focus. J Comput Assist Tomogr 2013;37:602-9.

Cite this article as: Bini R, Ronchetta C, Picotto S, Scozzari G, Gupta S, Frassini S, Chiara O. Importance of CT-scan predicting clinical outcomes in gastro-intestinal perforation. Ann Transl Med 2020;8(21):1421. doi: 10.21037/atm-20-2184 